\title{
Free Vascularized Fibular Graft for Congenital Pseudarthrosis Tibia: A Case Series
}

\author{
Nico Lie*, Anak Agung Gde Yuda Asmara** \\ *Resident Orthopaedic and Traumatology, ${ }^{* *}$ Hand and Microsurgery Consultant, \\ Faculty of Medicine Udayana University, Sanglah General Hospital, Denpasar, Bali \\ Corresponding Author: Nico Lie
}

\begin{abstract}
Introduction: Congenital pseudarthrosis tibia (CPT) probably one of the most difficult to treat among all diseases in the children. There are several surgical approaches that have been used to treat CPT including on-lay graft, double onlay grafts, pedicle grafts, osteotomy, bypass graft and intramedullary rods. Prognosis of CPT has changed considerably with the use of Free Vascularized Fibular Graft (FVFG). Despite these advances, several operations are often necessary to obtain union of CPT and the risk of amputation is never entirely eliminated
\end{abstract}

Case presentation: We presented 2 case with CPT. The first case is a 4 year old girl with CPT-Associated Neurofibromatosis, patient was brought to orthopedic polyclinic complaining bend on her right leg since she was born, the parent also complained her child has abnormality when walking since she was 14 months old. The second case is a 3 year old girl with CPT complaining bend on her right leg, abnormalities and pain when walking since she was aged 1 years 11 months. We performed free vascularized fibular graft for both of the patient.

Result: The results in our cases showed bone union in 14 weeks, 16 weeks and 18 weeks respectively. The optimal of technique options should be adapted to the type of pseudarthrosis and especially to the extent of the bone defects. Good results can be found with intramedullary nailing with a bone graft or the Ilizarov technique

Conclusion: The optimal of technique options should be adapted to the type of pseudarthrosis and especially to the extent of the bone defects.

Keywords: Congenital pseudarthrosis tibia, Free vascularized fibular graft, case series

\section{INTRODUCTION}

Congenital pseudarthrosis tibia (CPT) is probably one of the most difficult to treat among all diseases in the children. Nowadays, failure to obtain bone union is still common and the functional prognosis is in doubt because of residual deformities. $\mathrm{CPT}$ is related with type 1 neurofibromatosis even though the clear relation in between is unknown. ${ }^{1}$

Considered as a rare disease in children, CPT prevalence is 1 in 150.000 births. Some literatures defined this condition as a disorder of the diaphysis which is revealed by either pseudarthrosis at birth or a by pathological fracture presenting in the bone with bowing deformities, narrowing of the medullary canal or a cyst. ${ }^{1}$

The pathophysiology of CPT has still not been clearly defined. There are several surgical approaches that have been used to treat CPT including on-lay graft, double on-lay grafts, pedicle grafts, osteotomy, bypass graft and intramedullary rods. Nevertheless, early amputation has been advocated in several cases since the prognosis of such procedures are poor. ${ }^{1,2}$

Along with the development of microsurgery, successful attempts have been reported by using free vascularized bone graft to bridge the gap of the pseudarthrosis. Numerous mechanical and/ or biological based approaches have been described in many literatures to treat this condition with various successful rate. Prognosis of CPT has changed considerably with the use of 
Free Vascularized Fibular Graft (FVFG). Despite these advances, several operations are often necessary to obtain union of CPT and the risk of amputation is never entirely eliminated. ${ }^{2}$

\section{Anatomical Pathology}

There is a marked cuff of fibrous tissue in the area of pseudarthrosis in histological studies, also called fibrous hamartoma in continuity with abnormal periosteal thickening; most of the cell that identified in the hamartoma is fibroblasts. A certain fibrocartilage and hyaline cartilage is associated with the fibrous tissue as well. In addition to this, resorption lacunas with giant osteoclast are present at the pseudarthrosis-bone junction. This osteolytic component seems to be marked in young children and decreased with growth, then disappears at skeletal maturity. All of the above phenomena are sign of a bone remodeling defect which is responsible for the sclerotic, hourglass appearance of the bone adjacent to the pseudarthrosis as well as the atrophy of the bone ends. The composition of the pseudarthrotic tissue seems to be identical with NF1.,3

\section{Pathophysiology}

The etiology of CPT is still unclear. There are various mechanical, vascular or genetic theories that have been proposed in some literatures. None of the provide an entirely satisfying explanation for the pathogenesis of CPT or its location, probably because of the heterogeneity of the disease and its inconsistency association with NF 1.

\section{Surgical Technique ${ }^{4,5}$}

Before operation to outline the vascular anatomy of the donor and recipient legs, angiography was performed. This was important especially in those who had previous operations.

\section{Donor Site}

The length of the fibula for the graft was determined according to the length of the bone defect. The operation was carried out as described.

\section{Recipient Site (Excision of the Pseudarthrosis)}

The longitudinal anteromedial incision is performed to explore the pseudoarthrosis. All abnormal periosteum, soft tissue, and bone are completely excised as described previously. The entire sclerotic portion of the medullary canal and all abnormal tissue be removed. The fibular graft is inserted into the medullary canals in both cut ends of the recipient tibia. It is inserted into the proximal tibia fragment first then the distal fragment. Anastomosis was performed between the donor peroneal artery and the recipient anterior tibial artery and between the vena comitantes of the peroneal artery and the vena comitantes of the recipient anterior tibial artery using 9-0 or 10-0 nylon sutures under an operating microscope. Anastomoses between these vessels were successful in all cases. After the operation, surgically treated leg is immobilized with above-knee plaster backslab for 3 weeks. Later, above-knee cast is worn until bony union occurred.

\section{CASE PRESENTATION}

\section{Case 1}

A 4 year old girl with CPTAssociated Neurofibromatosis, patient was brought to orthopedic polyclinic complaining bend on her right leg since she was born, the parent also complained her child has abnormality when walking since she was 14 months old. When patient first came to polyclinic LLD measured at $2 \mathrm{~cm}$. The pseudarthrosis site was explored through a longitudinal incision of the anterior tibia. Both nonunion stumps of the tibia and the surrounding fibrous tissues were resected as minimally as possible. Longitudinal osteotomies of the tibia were performed, in the sagittal plane, the anterior and posterior cortical bone of the tibia were cut with a bone saw. A fibular section measuring $6 \mathrm{~cm}$ along with the peroneal artery and vena comitans was harvested 
from the contralateral leg. The periosteum was peeled at both ends of the graft, and the muscular soft tissue was resected on the surface contacting the tibial medulla. The harvested fibula with vascular pedicle was placed into the tibial. The fibular graft was fixed to the tibia with a screw at the distal end to obtain stability. After this, vascular anastomoses were performed without complications.
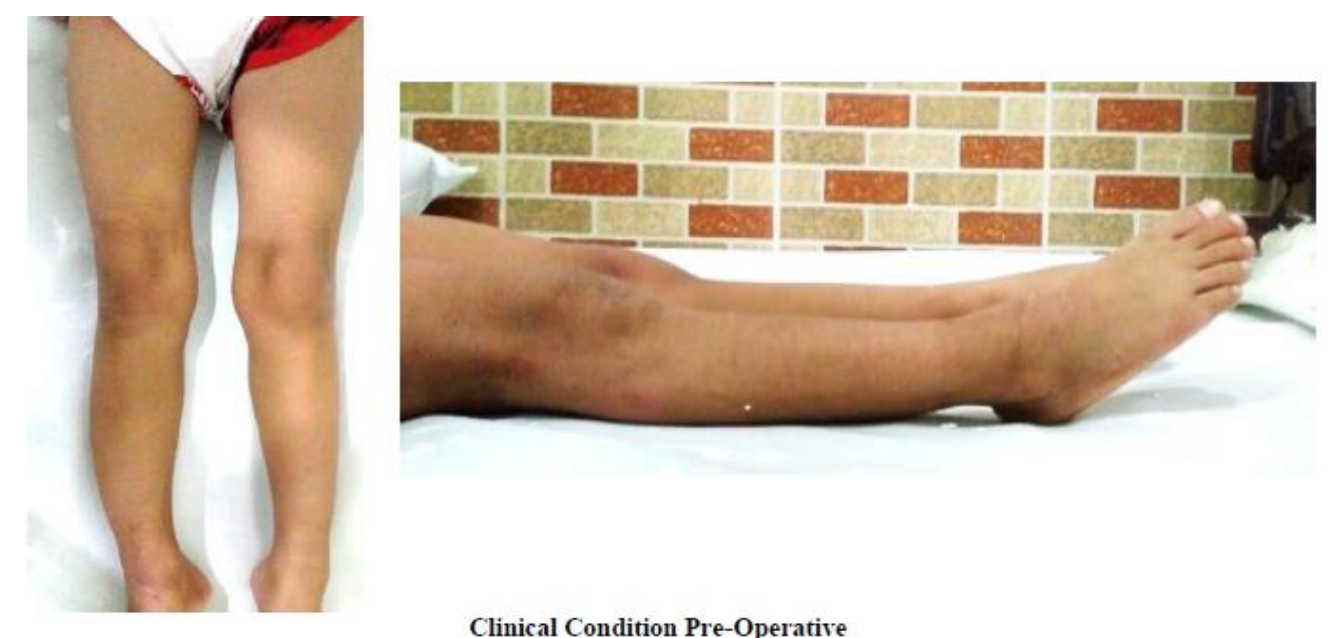

Clinical Condition Pre-Operative

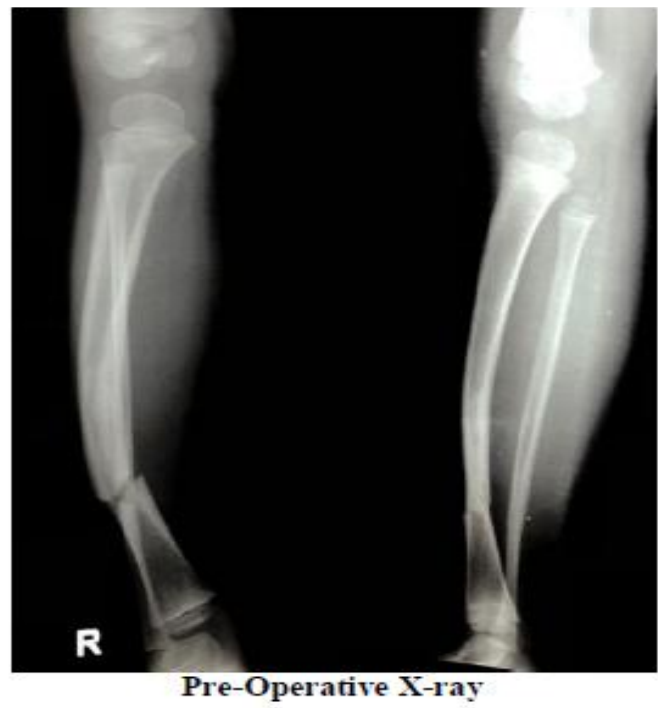

Post-operative LLD remain in $1 \mathrm{~cm}$ The range of motion (ROM) of the knee was 140 degrees of flexion and 0 degree of extension, and the ROM of the ankle was 15 degrees of dorsiflexion and 25 degrees of plantarflexion; all measurements were the same as those preoperatively and without any residual ankle pain.

\section{Case2}

A 3 year old girl with CPT patient was brought to orthopedic polyclinic complaining bend on her right leg since she was aged 1 years 11 months, the parent also complained her child has abnormalities when walking and also complaining pain while walking. Patient rarely standing with her own leg, she crawling when mobilize. LLD Measured $4 \mathrm{~cm}$ at first consultation. The surgery technique as described in case one with fibular graft measuring at $6 \mathrm{~cm}$ along with the peroneal artery and vena comitans was harvested from the contralateral leg.

Post-operative LLD remain in $2 \mathrm{~cm}$ The range of motion (ROM) of the knee was 140 degrees of flexion and 0 degree of extension, and the ROM of the ankle was 15 degrees of dorsiflexion and 35 degrees of plantarflexion; all measurements were the 
same as those preoperatively and without any residual ankle pain.
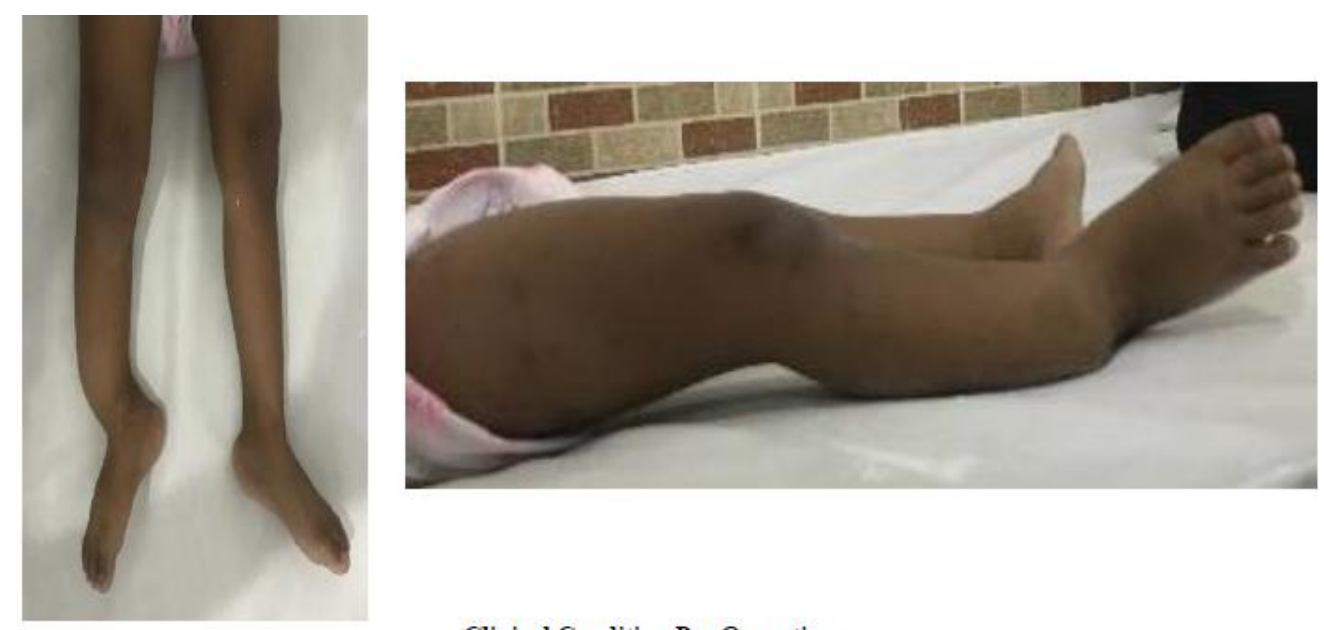

Clinical Condition Pre Operative

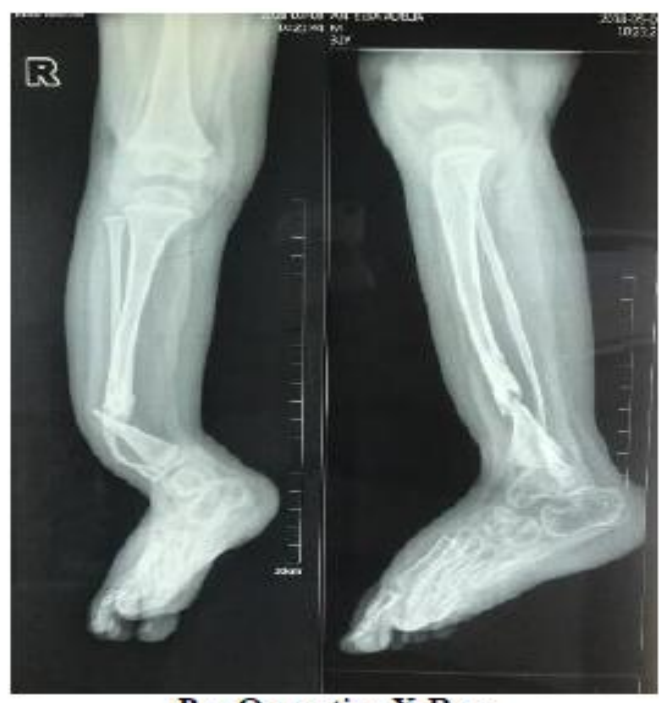

Pre Operative X-Ray
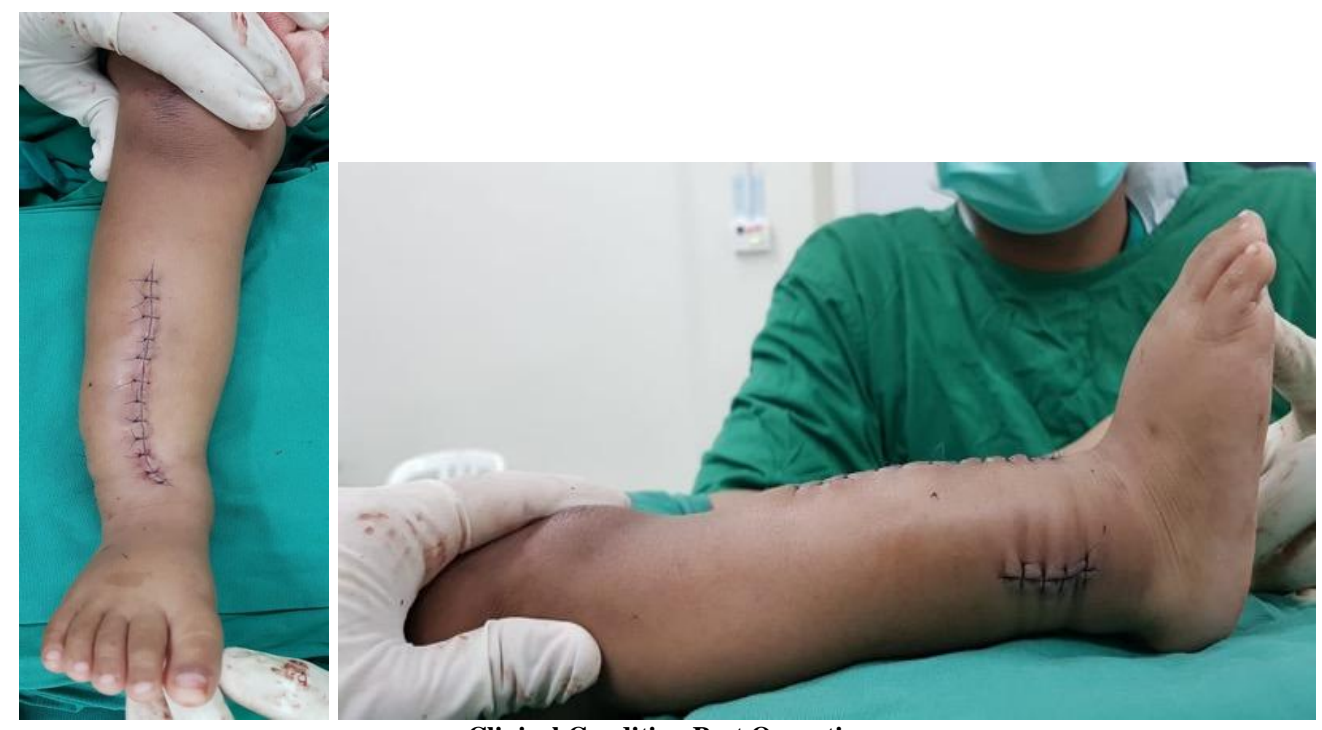

Clinical Condition Post Operative 


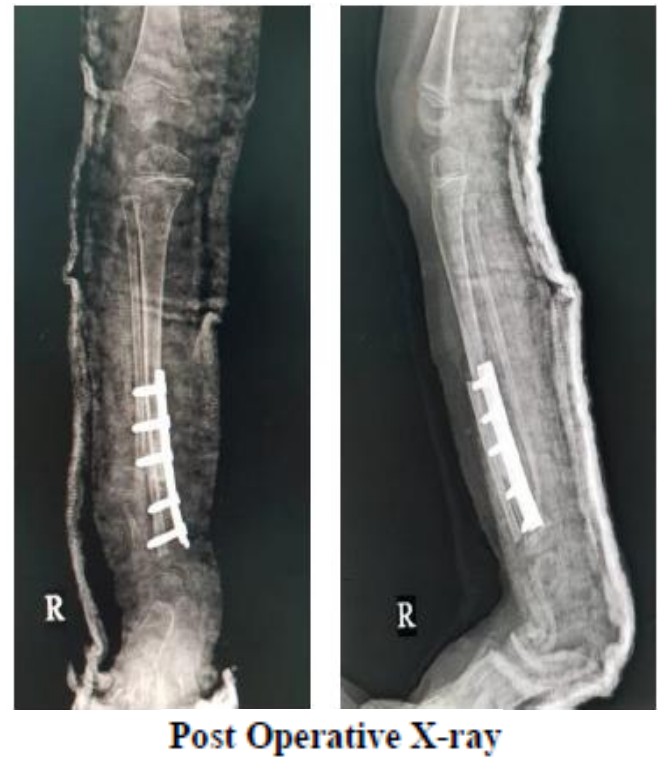

Case 3

A 3 years old boy with CPT was brought to orthopedic polyclinic complaining bend on her leg since she was aged 1 years 8 months, parents also complained her child has abnormalities when walking, patient had history of trauma on the left leg. LLD Measured $3 \mathrm{~cm}$ at first consultation. The surgery technique as described in case one with fibular graft measuring at $6 \mathrm{~cm}$ along with the peroneal artery and vena comitans was harvested from the contralateral leg.

Post operative LLD remain in $2 \mathrm{~cm}$ The range of motion (ROM) of the knee was 135 degrees of flexion and 0 degree of extension, and the ROM of the ankle was 15 degrees of dorsiflexion and 35 degrees of plantarflexion; all measurements were the same as those preoperatively and without any residual ankle pain.
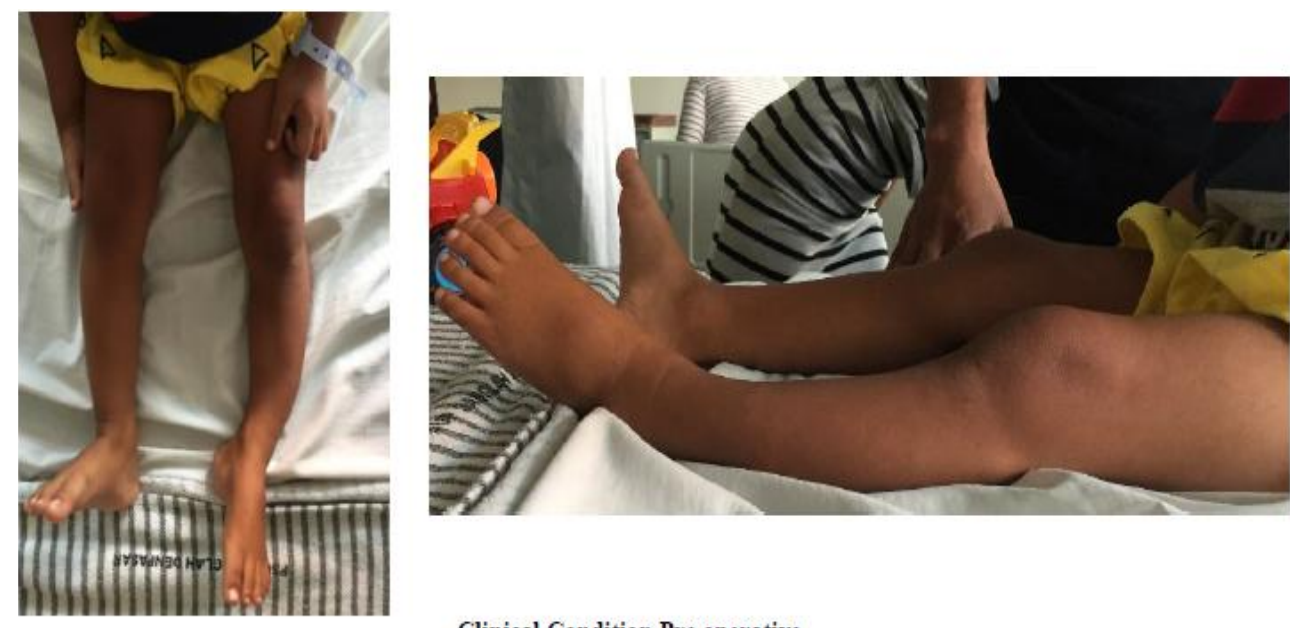

Clinical Condition Pre operative

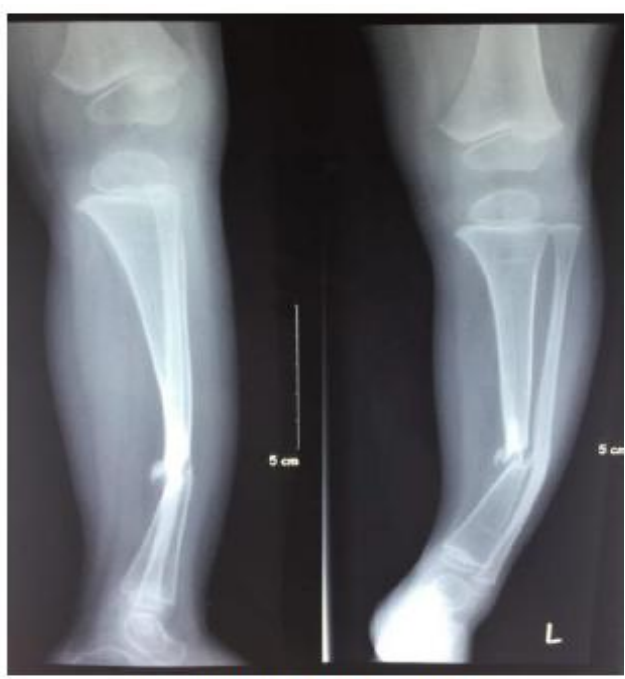

Pre operative Left Leg X-Ray

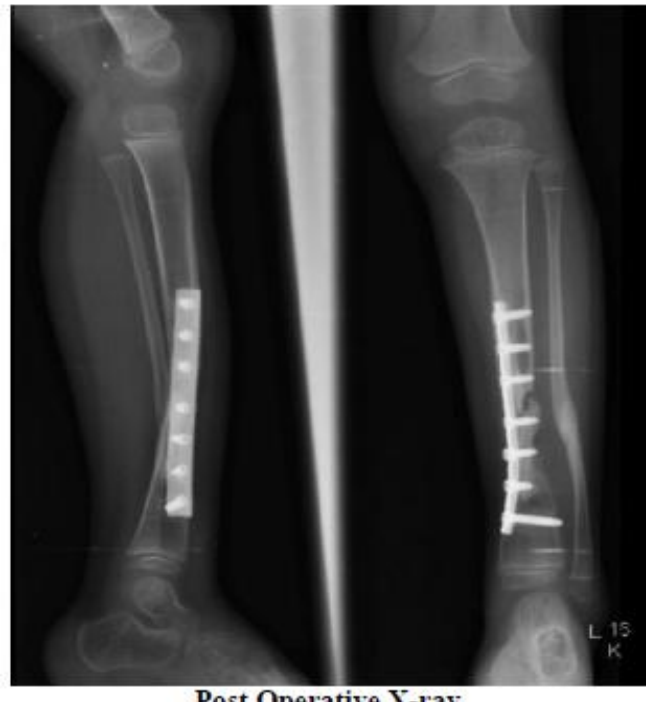

Post Operative X-ray 


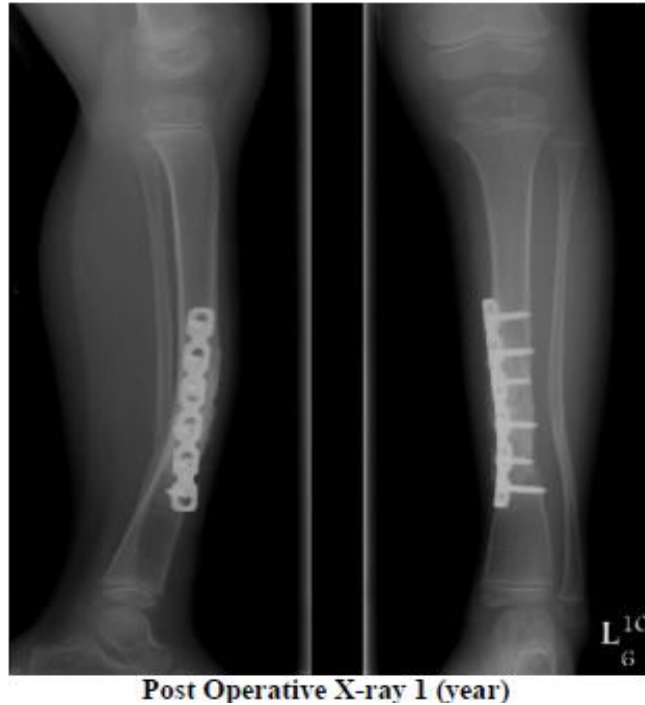

\section{DISCUSSION}

The results in our cases showed bone union in 14 weeks, 16 weeks and 18 weeks respectively. Previous studies suggested that a refracture was associated with a narrow bone diameter and longer fibular graft but there is no refractures occurred in our cases. Although there has been a report clarifying the correlation between refracture and diameter of the tibia, we believe that an increase in the contact area between the vascularized fibular graft and the recipient tibia promotes bone union, increases stability of the graft, enlarges the diameter of the graft, protects the tibia against deformity, and eventually results in the prevention of refracture. ${ }^{6}$

The etiopathogenesis of CPT is still controversial. Several previous studies have suggested that the formation of abnormal fibrous tissues around the pseudarthrosis causes a disturbance in blood circulation in the periosteum and ensuing its degeneration. This is in turn results in bone remodelling abnormalities and the advancement of pseudarthrosis. In conventional FVFG, the abnormal pseudarthrotic bone is resected radically and a fibular graft is placed into the vacant space. Uchida et al and Sakamoto et al reported the original technique in which an ipsilateral or contralateral fibular graft was fixed onto the side of the tibia after minimum resection of the sclerotic bone end and have obtained satisfactory results. This technique emphasized that the remaining sclerotic bone contributes to bone union subsequent to minimum resection, correction of alignment was feasible, and radical resection was not necessary. ${ }^{1,3}$

Even though best treatment for CPT remains controversial, there is a consensus on a certain number of points in some literatures. Whatever the technique being used, the tibial segment and stable internal fixation realignment are essential for union. The best technique for maintaining alignment during growth and for preventing the recurrent fracture risk is intramedullary fixation. The association of an intramedullary nail with the Illizarov technique is now being developed. This approach increases stability, preserves longterm alignment and/or guides lengthening although the risk of infection is increased,. The rate of recurrent fracture is reduced for more than $50 \%$ of cases in particular during ablation of the external fixator. Intramedullary fixation is more difficult with vascularized fibular transfer because of the risk of injury to the vascularized transplant. Axial deformities, which are the source of recurrent fracture, cannot be prevented with external fixation. The role of fibular surgery has probably been underestimated in some literatures. Indeed, persistent fibular pseudarthrosis favors valgus ankle and can explain the high rate of recurrent fractures after primary union. Treatment of the fibula is systematic in case of pseudarthrosis for many authors. Fibular union associated or not with internal fixation by intramedullary nailing reinforces stability by distributing loads and protects, in particular, from rotational trauma. If continuity is preserved or if the fibula is the site of hypoplasia or bowing, the choice of treatment is debatable. Some authors do not intervene, others perform fusion by intertibiofibular graft after tibial union. Finally, osteotomy to shorten the fibula is necessary for realignment. The purpose of this technique is to prevent distraction of the area of tibial pseudarthrosis by favoring the 
contact of the two bone ends and their union. 6,7

The indication for surgery is based on the child's age, family situation, the type and location of pseudarthrosis as well as the surgical history. EPOS recommends surgical management in children after the age of 3 because of the difficulty of stabilizing small bone fragments in younger children. These mechanical problems are supported by the biological concepts found in the studies showed that osteolytic activity in the area of pseudarthrosis was more important in the young child. ${ }^{3,5,7}$

Until recently, there is no gold standard surgical technique to successfully treat all types and presentations of CPT. The optimal of technique options should be adapted to the type of pseudarthrosis and especially to the extent of the bone defects. In normo and hypertrophic types, when there is little shortening. The debate commonly involves atrophic forms in which resection results in significant loss of bone substance. The limitation of this study is limited follow up duration and the patients' compliance to take routine controls (monthly). All the above 3 cases have reached the bony union yet further followups are needed until the bone maturity age is reach.

\section{CONCLUSION}

The optimal of technique options should be adapted to the type of pseudarthrosis and especially to the extent of the bone defects. Good results can be found with intramedullary nailing with a bone graft or the Ilizarov technique. An important role in this indication is vascularized fibula and the Ilizarov method with bone transport play. Some considerations include transfer of healthy vascularized bone in the first case and increasing blood flow by proximal corticotomy and diaphyseal transfer in the second, creates a favorable vascular environment for union while compensating for bone defects.

\section{Acknowledgement: None}

\section{Conflict of Interest: None}

\section{Source of Funding: None}

\section{REFERENCES}

1. Pannier S. Congenital pseudarthrosis of the tibia. Orthopaedics and Traumatology: Surgery and Research. 2011.

2. Takazawa A, Matsuda S, Fujioka F, Uchiyama S, Kato H. Split tibia vascularized fibular graft for congenital pseudarthrosis of the tibia: A preliminary report of 2 cases. J Pediatr Orthop. 2011;

3. Romanus B, Bollini G, Dungl P, Fixsen J, Grill F, Hefti F, et al. Free vascular fibular transfer in congenital pseudoarthrosis of the tibia: Results of the EPOS Multicenter Study. J Pediatr Orthop Part B. 2000;

4. Grill F, Bollini G, Dungl P, Fixsen J, Hefti $\mathrm{F}$, Ippolito $\mathrm{E}$, et al. Treatment approaches for congenital pseudarthrosis of tibia: Results of the EPOS Multicenter Study. J Pediatr Orthop Part B. 2000;

5. Ohnishi I, Sato W, Matsuyama J, Yajima H, Haga N, Kamegaya M, et al. Treatment of congenital pseudarthrosis of the tibia: A multicenter study in Japan. J Pediatr Orthop. 2005;

6. Sakamoto A, Yoshida T, Uchida Y, Kojima $\mathrm{T}$, Kubota $\mathrm{H}$, Iwamoto $\mathrm{Y}$. Long-term follow-up on the use of vascularized fibular graft for the treatment of congenital pseudarthrosis of the tibia. J Orthop Surg Res. 2008;

7. Joseph B, Somaraju VVJ, Shetty SK. Management of Congenital Pseudarthrosis of the Tibia in Children Under 3 Years of Age: Effect of Early Surgery on Union of the Pseudarthrosis and Growth of the Limb. J Pediatr Orthop. 2003;

How to cite this article: Nico Lie, Anak Agung Gde Yuda Asmara. Free Vascularized Fibular Graft for congenital pseudarthrosis tibia: a case series. International Journal of Science \& Healthcare Research. 2021; 6(3): 315-321. DOI: https://doi.org/10.52403/ijshr.20210754 